PROCEEDINGS OF THE

AMERICAN MATHEMATICAL SOCIETY

Volume 138, Number 11, November 2010, Pages 4123-4128

S 0002-9939(2010)10466-5

Article electronically published on July 7, 2010

\title{
BARBASHIN-KRASOVSKII THEOREM FOR STOCHASTIC DIFFERENTIAL EQUATIONS
}

\author{
OLEKSIY IGNATYEV AND V. MANDREKAR
}

(Communicated by Richard C. Bradley)

\begin{abstract}
A system of stochastic differential equations $d X(t)=f(X) d t+$ $\sum_{i=1}^{k} g_{i}(X) d W_{i}(t)$ which has a zero solution $X=0$ is considered. It is assumed that there exists a positive definite function $V(x)$ such that the corresponding operator $L V$ is nonpositive. It is proved that if the set $\{M: L V=0\}$ does not include entire semitrajectories of the system almost surely, then the zero solution is asymptotically stable in probability.
\end{abstract}

\section{INTRODUCTION}

The main method for investigating the stability and asymptotic stability in probability of the zero solution of the systems of stochastic differential equations is Lyapunov's Direct (or Second) Method.

Lyapunov's initial results and the initial work involving Lyapunov vectorfunctions pertained to finite-dimensional dynamical systems determined by ordinary differential equations. However, it was quickly recognized that the Second Method can be extended to also be applicable to finite-dimensional dynamical systems determined by difference equations and to infinite-dimensional dynamical systems defined on Hilbert, Banach and even general metric spaces. (Such dynamical systems are determined, e.g., by functional differential equations, partial differential equations, Volterra integro-differential equations and others.)

Very often, when we are interested in investigation of asymptotic stability of the zero solution, in applications it is possible to construct the Lyapunov function when its derivative is nonpositive only. In this case Barbashin and Krasovskii proved a well-known result [2] on asymptotic stability. This result was extended in [9, [10] and [11] to the case of periodic and almost periodic systems.

Has'minskii [7, 8, proved a generalization of Lyapunov theorems in the case of stochastic differential equations. Has'minskii also proved a theorem 8 on asymptotic stability in probability using the nondegeneracy condition requirement for the matrix, corresponding to the coefficients of Wiener processes. Many results concerning the stability and asymptotic stability in probability of the zero solution of stochastic differential equations were obtained also by Kushner [14, 15, Mao

Received by the editors August 7, 2009 and, in revised form, February 27, 2010.

2010 Mathematics Subject Classification. Primary 60H10, 93E15.

Key words and phrases. Stochastic differential equations, Lyapunov functions, asymptotic stability in probability.

(C)2010 American Mathematical Society Reverts to public domain 28 years from publication 
[16. 17 and others. In recent years the method of Lyapunov's functions for stochastic differential equations has been developed in [1, 3, 4, 5, 12, 13, 18, 19] and others.

In this paper, we obtain the criteria of the asymptotic stability in probability of the zero solution of a system of stochastic differential equations in the case when $L V \leq 0$ and the set $\{M: L V=0\}$ does not include entire semitrajectories of the system (2.1) almost surely. This result generalizes the Barbashin-Krasovskii theorem [2] to the case of stochastic differential equations.

\section{Definitions AND PRELIMINARY RESUlts}

Consider the system of autonomous stochastic differential equations

$$
d X(t)=f(X) d t+\sum_{i=1}^{k} g_{i}(X) d W_{i}(t), \quad f(0) \equiv 0, \quad g_{i}(0) \equiv 0,
$$

where $X=\left(X_{1}, \ldots, X_{n}\right) \in R^{n}, t \in[s,+\infty)$ is time. We assume that $f(X), g_{i}(X)$ are vectors in $R^{n}$ and $W_{i}(t)$ are independent Wiener processes. We assume that the functions $f(X)$ and $g_{i}(X)$ satisfy the Lipschitz condition in $X$ in every bounded in $X$ domain, i.e.,

$$
\sum_{i=1}^{k}\left|g_{i}(x)-g_{i}(y)\right|+|f(x)-f(y)|<C|x-y| .
$$

$$
\begin{aligned}
& \text { Denote }\|X\|=\sqrt{X_{1}^{2}+\cdots+X_{n}^{2}} \text { and } \\
& \qquad B_{H}:=\left\{x \in R^{n}:\|x\|<H\right\} .
\end{aligned}
$$

Denote by $X^{s, x}(t)$ the solution of (2.1) with initial condition $X^{s, x}(s)=x$. Let us define the $n \times k$ matrix $g(x)=\left(g_{1}(x), \ldots, g_{k}(x)\right)$ and the $n \times n$ matrix $A(x)=$ $\left[a_{i j}(x)\right]=g(x) g^{T}(x)$, where $g^{T}(x)$ is a transpose matrix of $g(x)$. Set $\tau_{S_{H}}(t)=$ $\min \left(\tau_{S_{H}}, t\right)$, where $\tau_{S_{H}}$ is the first exit time from the $B_{H}$ of the sample path of the process $X(t)$ defined by equation $(2.1)$.

Let

$$
L V(x)=\sum_{i=1}^{n} f_{i}(x) \frac{\partial V}{\partial x_{i}}+\frac{1}{2} \sum_{i, j=1}^{n} a_{i j}(x) \frac{\partial^{2} V}{\partial x_{i} \partial x_{j}} .
$$

Definition 2.1. The trivial solution of system (2.1) is said to be stable in probability if for every $\varepsilon>0$ and $s \in R_{+}$

$$
\lim _{x \rightarrow 0} P\left\{\sup _{t>s}\left\|X^{s, x}(t)\right\|>\varepsilon\right\}=0 .
$$

Definition 2.2. The trivial solution of system (2.1) is said to be asymptotically stable in probability if it is stable in probability and

$$
\lim _{x \rightarrow 0} P\left\{\lim _{t \rightarrow \infty}\left\|X^{s, x}(t)\right\|=0\right\}=1 .
$$

Definition 2.3. According to [6, 21] we say that a function $g: R_{+} \rightarrow R_{+}$is Hahn's function $(g \in \mathcal{K})$ if it is continuous, monotonically increasing and $g(0)=0$.

Definition 2.4 (21]). A function $V: R^{n} \rightarrow R$ is said to be positive definite if there exists $\alpha \in \mathcal{K}$ such that $V(x) \geq \alpha(\|x\|)$.

Definition 2.5 (21]). A function $V: R^{n} \rightarrow R$ is said to have an infinitesimal upper limit if there exists $\beta \in \mathcal{K}$ such that $V(x) \leq \beta(\|x\|)$. 
Lemma 2.1 ([8]). Let $V(x)$ be a function in class $C_{2}^{0}\left(B_{H}\right)$, bounded in the domain $B_{H}$, and suppose that $L V(x) \leq 0$ in this domain. Then the process $V\left(X^{s, x}\left(\tau_{S_{H}}(t)\right)\right)$ is a supermartingale, so that

$$
E V\left(X^{s, x}\left(\tau_{S_{H}}(t)\right)\right) \leq V(x)
$$

for $x \in B_{H}$.

Theorem $2.1([8])$. Assume that in the domain $B_{H}$ there exists a positive definite function $V(x) \in C_{2}^{0}\left(B_{H}\right)$ which satisfies the condition

$$
L V \leq 0
$$

for $x \neq 0$. Then the trivial solution $X(t) \equiv 0$ of the system (2.1) is stable in probability.

Theorem 2.2 ([8]). If in the domain $B_{H}$ there exists a function $V(x) \in C_{2}^{0}\left(B_{H}\right)$ which satisfies the following conditions:

(1) $\alpha(\|x\|) \leq V(x) \leq \beta(\|x\|)$,

(2) $L V(x) \leq-\gamma(\|x\|)$,

where $\alpha, \beta, \gamma \in \mathcal{K}$, then the trivial solution $X(t) \equiv 0$ of system (2.1) is asymptotically stable in probability.

\section{MAIN RESUlts}

Theorem 3.1. Suppose that there exists a positive definite function $V(x) \in C_{2}^{0}\left(B_{H}\right)$ such that $L V$ satisfies almost surely the following conditions in the domain $B_{H} \backslash\{0\}$ :

(1) $L V<0$ outside of the set $M$,

(2) $L V=0$ on $M$,

where the set $M$ does not include entire semitrajectories $X^{s, x}(t)$ of the system (2.1) for $s<t<\infty$ (with the exception of $X \equiv 0$ ). Then the zero solution of equations (2.1) is asymptotically stable in probability.

Proof. Fix any $\varepsilon>0$. Let $K_{x}$ denote the set of sample paths of $X^{s, x}(t)$ such that $\tau_{S_{\varepsilon}}=\infty$. Since the function $V$ satisfies the assumptions of Theorem 2.1, the solution $X(t) \equiv 0$ is stable in probability, and therefore

$$
P\left(K_{x}\right) \rightarrow 1, \quad \text { as } \quad x \rightarrow 0 .
$$

In other words,

$$
P\left(\left\|X^{s, x}(t)\right\|<\varepsilon\right) \rightarrow 1, \quad \text { as } \quad x \rightarrow 0 \quad \text { for all } \quad \varepsilon>0 .
$$

Let us show that the solution $X(t) \equiv 0$ is asymptotically stable in probability. Since $L V \leq 0$, using Lemma 2.1 we have

$$
V_{1}\left(X^{s, x}(t)\right):=E V\left(X^{s, x}\left(\tau_{S_{H}}(t)\right)\right) \leq V(x) \text { for all } t \geq s,
$$

and the deterministic function $V_{1}\left(X^{s, x}(t)\right)$ is a nonnegative, bounded function of $t$. Since $L V \leq 0$, Lemma 3.3.1 from [8] implies that the function $V_{1}\left(X^{s, x}(t)\right)$ is also a nonincreasing function of $t$. Therefore this function has some limit $V_{0}$ as $t \rightarrow \infty$; moreover

$$
V_{1}\left(X^{s, x}(t)\right) \geq V_{0} \quad \text { for } \quad t \geq s .
$$

Suppose that $V_{0} \neq 0$. Using (3.2) and the fact that area $\left\|X^{s, x}(t)\right\|<\varepsilon$ is bounded, we can conclude that there exists a sequence of points $X_{(k)}^{s, x}=X^{s, x}\left(s+k t_{1}\right)(k=$ $k_{1}, k_{2}, \ldots ; t_{1}=$ const $>0$ ) which is convergent almost surely to the point $q$, with 
coordinates $X^{*}=\left(X_{1}^{*}, X_{2}^{*}, \ldots, X_{n}^{*}\right)$, belonging to the area $\left\|X^{s, x}(t)\right\| \leq \varepsilon$. Using the continuity of the function $V_{1}$ we have $V_{1}\left(X_{1}^{*}, X_{2}^{*}, \ldots, X_{n}^{*}\right)=V_{0}$.

Consider now the solutions of $(2.1) X^{s, q}\left(\tau_{S_{\varepsilon}}(t)\right)$ and $X^{s, k_{i}}\left(\tau_{S_{\varepsilon}}(t)\right)$, starting at moment $t=s$ from the points $q$ and $X_{\left(k_{i}\right)}^{s, x}$ respectively. Since the solution $X^{s, q}\left(\tau_{S_{\varepsilon}}(t)\right)$ for $s \leq t<\infty$ almost surely does not belong to the set $M$ completely, there should exist some time interval when $L V<0$ along this solution. This means, using Lemma 3.3.1 from [8], that there exists a time moment $T>s$ such that

$$
V_{1}\left(X^{s, q}(T)\right)=V_{2}<V_{0} .
$$

Since the sequence $X^{s, k_{i}}\left(\tau_{S_{\varepsilon}}(t)\right)$ converges to the point $q$ with probability 1 , according to [20] we have the inequality

$$
E\left(X^{s, q}(T)-X^{s, k_{i}}(T)\right)^{2} \leq C E\left\|X^{*}-X^{s, x}\left(s+k_{i} t_{1}\right)\right\|^{2} \rightarrow 0 \quad \text { as } \quad k_{i} \rightarrow \infty,
$$

where $C=$ const $>0$. Hence, we have

$$
E\left(X^{s, q}(T)-X^{s, k_{i}}(T)\right)^{2} \rightarrow 0 \quad \text { as } \quad k_{i} \rightarrow \infty .
$$

Using the stability in probability of the solutions $X^{s, q}(t)$ and $X^{s, k_{i}}(t)$, we see that $\left(X^{s, q}(T)-X^{s, k_{i}}(T)\right)^{2}$ is nonnegative and bounded, and by Skorokhod [22], there exists a probability space $\left(\Omega^{s}, F^{s}, P^{s}\right)$ such that almost surely $P^{s}$ we have

$$
\left(X^{s, q}(T)-X^{s, k_{i}}(T)\right)^{2} \rightarrow 0 \quad \text { as } \quad k_{i} \rightarrow \infty .
$$

Therefore, using (3.4) and continuity of the function $V_{1}$, we have

$$
\lim _{k_{i} \rightarrow \infty} V_{1}\left(X^{s, k_{i}}(T)\right)=V_{2} .
$$

Since the functions $f(X)$ and $g_{i}(X)$ in (2.1) are independent of time $t$, on the probability space $\left(\Omega^{s}, F^{s}, P^{s}\right)$ with probability 1 we have

$$
X^{s, k_{i}}(T)=X^{s, x}\left(T+s+k_{i} t_{1}\right)
$$

hence we can rewrite (3.5) in the following form:

$$
\lim _{k_{i} \rightarrow \infty} V_{1}\left(X^{s, x}\left(T+s+k_{i} t_{1}\right)\right)=V_{2}<V_{0} .
$$

But (3.6) contradicts (3.3); therefore our assumption that $V_{0} \neq 0$ is wrong, and hence $V_{0}=0$. This means that

$$
\lim _{t \rightarrow \infty} V_{1}\left(X^{s, x}(t)\right)=0 .
$$

Hence

$$
\lim _{t \rightarrow \infty}\left\|X^{s, x}(t)\right\|=0
$$

with probability 1 .

Example 3.1. Consider the system of stochastic differential equations

$$
\begin{aligned}
& d y=-y\left(z^{2}+1\right) d t+y \sqrt{z^{2}+1} d W_{1}(t), \\
& d z=-z d t+y d W_{2}(t) .
\end{aligned}
$$

This system has the trivial solution

$$
y=0, \quad z=0 .
$$

Let us show that this solution is asymptotically stable in probability. Denote

$$
V:=\frac{1}{2}\left(y^{2}+z^{2}\right) \text {. }
$$


We get

$$
L V=-y^{2}\left(z^{2}+1\right)+\frac{1}{2} y^{2}\left(z^{2}+1\right)-z^{2}+\frac{1}{2} y^{2}=-z^{2}\left(\frac{1}{2} y^{2}+1\right) \leq 0 .
$$

Note that we cannot apply Theorem 2.2, because condition 2 of this theorem is not fulfilled. But we can apply Theorem 3.1 , since $L V=0$ on the set $M=\{(y, z)$ : $z=0\}$. We see from the second equation of (3.7) that the set $M$ does not contain almost surely any nontrivial solution of the system (3.7). Therefore, solution (3.8) of system (3.7) is asymptotically stable in probability.

\section{REFERENCES}

[1] L. Arnold and B. Schmalfuss, Lyapunov's second method for dynamical systems. Journal of Differential Equations, 177 (2001) 235-265. MR.1867618(2002h:37093)

[2] E.A. Barbashin and N.N. Krasovskyii, On the stability of motion on the large. Dokl. Akad. Nauk USSR, 86 (1952) 453-456. (Russian) MR0052616 (14:646f)

[3] A. Cesaroni, A converse Lyapunov theorem for almost sure stabilizability. Systems \& Control Letters, 55 (2006) 992-998. MR2267391(2007i:93052)

[4] P. Florchinger, Lyapunov-like techniques for stochastic stability. SIAM J. Control and Optimization, 33 (1995) 1151-1169. MR1339059 (96d:93084)

[5] P. Florchinger, Feedback stabilization of affine in the control stochastic differential equations by the control Lyapunov function method. SIAM J. Control and Optimization, 35 (1997) 500-511. MR.1436635 (97k:93068)

[6] W. Hahn, Stability of Motion. Springer, New York - Berlin - Heidelberg, 1967. MR0223668 $(36: 6716)$

[7] R.Z. Has'minskii, On the stability of the trajectory of Markov processes. J. Appl. Math. Mech. 26 (1962) 1554-1565. MR0162271 (28:5470)

[8] R.Z. Has'minskii, Stochastic stability of differential equations. Sijthoff and Noordhoff, Rockville, Maryland, 1980. MR600653 (82b:60064)

[9] A.A. Ignat'ev, On equi-asymptotic stability with respect to some of the variables. J. Appl. Math. Mech, 63 (1999), 821-824. MR1754131(2001g:34065)

[10] A.A. Ignat'ev, Equiasymptotic stability of almost periodic systems. (Russian) Dopov. Nats. Akad. Nauk Ukr. Mat. Prirodozn. Tekh. Nauki, 10 (1997), pp. 32 - 35. MR1672971 (2000c:34127)

[11] A.O. Ignatyev, On the stability of equilibrium for almost periodic systems. Nonlinear Analysis. Theory, Methods and Applications, 29 (1997), 957-962. MR.1454820 (98j:34101)

[12] O. Ignatyev, Partial asymptotic stability in probability of stochastic differential equations. Statistics and Probability Letters, 79 (2009), 597-601. MR2499382

[13] V. Kolmanovskii and L. Shaikhet, Construction of Lyapunov functionals for stochastic hereditary systems: a survey of some recent results. Mathematical and Computer Modeling, 36 (2002), 691-716. MR1940617 (2003m:39020)

[14] H.J. Kushner, On the construction of stochastic Liapunov functions. IEEE Trans. Automatic Control, AC-10 (1965) 477-478.

[15] H.J. Kushner, Stochastic stability and control. Academic Press, New York, 1967. MR0216894 $(35: 7723)$

[16] X. Mao, Exponential stability for nonlinear stochastic differential equations with respect to semimartingales. Stochastics and Stochastics Reports, 28 (1989), 343-355. MR 1028538 (91g:60072)

[17] X. Mao, Exponential stability of stochastic differential equations. Marcel Dekker, New YorkBasel-Hong Kong, 1994. MR1275834 (95k:60153)

[18] X. Mao, Stochastic version of the LaSalle theorem. Journal of Differential Equations, 153 (1999), 175-195. MR1682267 (2000e:60094)

[19] X. Mao, Some contributions to stochastic asymptotic stability and boundedness via multiple Lyapunov functions. Journal of Mathematical Analysis and Applications, 260 (2001), 325-340. MR:1845557 (2002f:60119)

[20] B. Oksendal, Stochastic differential equations: an introduction with applications. Springer, Berlin-New York, 1998. MR,1619188(99c:60119) 
[21] N. Rouche, P. Habets, and M. Laloy, Stability Theory by Liapunov's Direct Method. SpringerVerlag, New York, 1977. MR0450715 (56:9008)

[22] A.V. Skorokhod, Studies in the theory of random processes. Addison-Wesley Publishing, Massachusetts, 1965. MR0185620(32:3082b)

Department of Statistics and Probability, Michigan State University, A408 Wells Hall, East Lansing, Michigan 48824-1027

E-mail address: ignatyev@stt.msu.edu

Department of Statistics and Probability, Michigan State University, East Lansing, MichigAn 48824-1027

E-mail address: mandrekar@stt.msu.edu 